EESTI NSV TEADUSTE AKADEEMIA TOIMETISED. FOUSIKA * MATEMAATIKA ИЗВЕСТИЯ АКАДЕМИИ НАУК ЭСТОНСКОИ ССР. ФИЗИКА * МАТЕМАТИКА PROCEEDINGS OF THE ACADEMY OF SCIENCES OF THE ESTONIAN SSR. PHYSICS * MATHEMATICS

1984, 33, 1

\title{
ПЕРЕХОДНЫЕ ПРОЦЕССЫ В СИЛОВЫХ ДИОДАХ НА ОСНОВЕ АРСЕНИДА ГАЛЛИЯ
}

Одно из важнейших свойств полупроводниковых приборов на основе арсенида галлия - их высокое быстродействие. При работе этих приборов в высокочастотных преобразовательных устройствах существенное значение приобретают коммутационные процессы переключения структур. Как показывают оценочные расчеты, длительность процесса коммутации у арсенид-галлиевых диодов вследствие малых времен жизни носителей заряда составляет десятые доли микросекунды [ $\left.{ }^{1}\right]$, что более чем ка порядок ниже, чем у кремниевых. Вместе с тем при разработке силовых диодов на основе арсенида галлия необходимы детальный количественный анализ и экспериментальные исследования их переходных процессов и частотных нагрузочных характеристик.

\section{1. Математическая модель и анализ переходного процесса установления стационарного состояния в силовых арсенид-галлиевых диодах}

Задача этого раздела настоящей работы - построение математической модели и исследование переходного процесса установления стационарного состояния в реальных диодных структурах на основе арсенида галлия с учетом основных нелинейных факторов: снижения эффективности эмиттерных переходов, влияния нагрузки, а также эффектов, присущих прямозонным полупроводникам. Такого исследования, как показал анализ литературных данных, до настоящего времени не проводилось.

Процесс установления стационарного состояния будем рассматривать на примере диодной структуры типа $P+-N-N^{+}$при следующих основных предположениях: 1) все области структуры легированы однородно; 2) времена жизни носителей заряда, подвижности и коэффициенты диффузии не зависят от координаты в пределах каждой из областей; 3) имеет место условие квазинейтральности; 4) уровень инжекции в базовой $N$-области полагается высоким, а в $P^{+}$- и $N^{+}$-эмиттерах низким.

Прй анализе электрофизических процессов в структурах на основе прямозонных полупроводников, каковым является арсенид галлия, необходим учет излучательного канала рекомбинации и генерации носителей за счет поглощения межзонного рекомбинационного излучения $\left[{ }^{4}\right]$.

Поскольку времена жизни в сильнолегированных эмиттерных областях на несколько порядков меньше, чем в слаболегированной базе, процессы в эмиттерах можно считать квазистационарными по отношению к 
процессам в $N$-базе. В таком случае задача может быть сформулирована только для базовой области структуры.

Нестационарное уравнение непрерывности для распределения концентрации дырок $p=p(x, t)$ в слаболегированной базе при высоком уровне инжекции $\left[^{2}\right]$ с учетом выражения для скорости излучательной рекомбинации $\left[{ }^{3}\right]$ и генерационного члена может быть записано в виде:

$$
\frac{2 b}{b+1} D \frac{\partial^{2} p}{\partial x^{2}}-\frac{p}{\tau_{N R}}-(1-v) B p\left(p+N_{d}\right)=\frac{\partial p}{\partial t},
$$

где $D$ - коэффициент диффузии дырок, $b$ - отношение подвижностей электронов и дырок, $N_{d}$ - концентрация доноров в $N$-базе, $\tau_{N R}-$ безызлучательное время жизни; $B=7,21 \cdot 10^{-10} \mathrm{~cm}^{3} / \mathrm{c}-$ коэффициент излучательной рекомбинации, $v-$ коэффициент фотонной генерации $\left[{ }^{4}\right]$.

Эффективное время жизни $\tau$ представляется в следующей форме $\left[{ }^{5}\right]$ :

$$
\tau^{-1}=\tau_{N R}^{-i}+(1-v) \tau_{R}^{-1}, \quad \tau_{R}^{-1}=B\left(p+N_{d}\right),
$$

где $\tau_{R}$ - излучательное время жизни. Учитывая наше предположение о независимости электрофизических параметров от координаты в каждой области $P^{+}-N-N^{+}$-структуры, определим усредненное эффективное время жизни носителей в пределах базовой области структуры толщиной $W$

$$
\tau(t)=\frac{1}{W} \int_{0}^{W}\left[\tau_{N R}^{-1}+(1-v) B\left(p(x, t)+N_{d}\right)\right]^{-1} d x
$$

Уравнение (1) тогда приводится к виду, формально идентичному уравнению непрерывности для дырок при учете только линейной рекомбинации через примесные центры (рекомбинация Шокли-Рида-Холла)

$$
\frac{\partial^{2} p}{\partial x^{2}}-\frac{p}{L^{2}}=\frac{\tau}{L^{2}} \frac{\partial p}{\partial t},
$$

в котором эффективное время жизни носителей $\tau$ и определяемая им диффузионная длина $L=\sqrt{\frac{2 b}{b+1} D \tau}$ нелинейно зависят от времени через нестационарную концентрацию дырок $p(x, t)$ в соответствии с формулой (3). Начальное условие может быть произвольным: $p(x, 0)=$ $=\varphi(x)$. Граничные условия задачи, учитывающие снижение эффективности эмиттерных переходов, а также уравнение электрического баланса напряжений внешней цепи формулируются точно так же, как и при анализе процесса установления в диодных (тиристорных) структурах на основе кремния, проведенном в $\left.{ }^{6}\right]$. При этом вид уравнения (4) позволяет, применяя метод квазилинеаризации $\left[{ }^{6}\right]$, использовать те же представления решений краевой задачи, что и в. более простом случае исследования процессов в приборах на основе непрямозонных полупроводников.

Разбивӑя весь переходный процесс на малые временные интервалы $\Delta t_{m}=t_{m+1}-t_{m} \ll \tau$ и линеаризуя задачу, будем полагать плотность тока $j$, коэффициенты инжекции эмиттерных переходов $\gamma_{1}, \gamma_{2}$ и эффективное время жизни постоянными в пределах каждого интервала. Тогда процесс установления при активной нагрузке описывается системой рекуррентных соотношений, приведенных в [ $\left.{ }^{6}\right]$, к которым' в нашем случае учета эффектов излучательной рекомбинации и фотонной генерации следует добавить рекуррентную формулу, описывающую изменение на каждом временном интервале эффективного времени жизни 


$$
p_{m+1}(x, t)=\alpha_{m+1}\left(x, t-t_{m}, j_{m}\right)+\int_{0}^{W} p_{m}\left(\xi, t_{m}\right) \beta_{m}\left(x, \xi, t-t_{m}\right) d \xi,
$$

$$
\begin{gathered}
\gamma_{1, m+1}=1-\frac{1}{j_{m}} h_{1} p_{m}^{2}\left(0, t_{m}\right) \\
\gamma_{2, m+1}=1-\frac{1}{j_{m}} h_{2} p_{m}^{2}\left(W, t_{m}\right) \\
j_{m}=\frac{U_{A}-U\left(t_{m}, j_{m}, p_{m}\right)\left(x, t_{m}, j_{m}\right)}{R S_{A}}, \\
\tau_{m+1}=\frac{1}{W} \int_{0}^{W}\left[\tau_{N R}^{-1}+(1-v) B\left(p_{m}\left(x, t_{m}\right)+N_{d}\right)\right]^{-1} d x \\
L_{m+1}=\sqrt{\frac{2 b}{b+1} D \tau_{m+1}}
\end{gathered}
$$

В этих формулах $p(0, t), p(\mathbb{W}, t)$ - граничные концентрации дырок у $P+-N$ - и $N^{+}-N$-переходов, $U_{A}-$ э.д.с. источника напряжения, $U-$ напряжение на диодной структуре, $R$ - активное сопротивление цепи, $S_{A}$ - площадь $P-N$-перехода. Развернутые выражения для $\alpha(x, t)$, $\beta(x, \xi, t), h_{1}, h_{2}, U(t)$ даны в $\left[{ }^{6}\right]$. Входящие в выражение $U(t)$ коэффициенты, описывающие рассеяние носителей на носителях в базе арсенидгаллиевой диодной структуры, приведены в $\left[{ }^{7}\right]$. Начальное распределение носителей на первом шаге зависит от начального состояния структуры и в общем может быть произвольным. Если к моменту подачи импульса напряжения заряд избыточных дырок в базе отсутствует, полагаем $p_{0}\left(x, t_{0}\right)=0$.

Перейдем к анализу результатов расчета. На рис. 1 представлен расчет характеристик переходного процесса установления стационарного состояния для различных значений коэффициента фотонной генерации. Параметры структуры и внешней цепи следующие: концентрация акцепторов в $P^{+}$- и $N^{+-}$-миттерах $N_{A}^{+}=10^{18} \mathrm{~cm}^{-3}, N_{d}^{+}=5 \cdot 10^{18} \mathrm{~cm}^{-3}$ соответственно; концентрация доноров в $N$-базе $N_{d}=10^{15}$ см; полагалось, что эффективные времена жизни в сильнолегированных эмиттерных областях определяются процессами безызлучательной рекомбинации, а их величины приняты равными $\tau_{N R}^{P^{*}}=10^{-10} \mathrm{c}, \quad \tau_{N R}^{N^{+}}=10^{-10} \mathrm{c} ;$ безызлучательное время жизни носителей в $N$-базе $\tau_{N R}=2 \cdot 10^{-7}$ с; коэффициент диффузии электронов в $P^{+}$- и дырок в $N$ - и $N^{+}$-областях $D_{n} P^{+}=80 \mathrm{~cm}^{2} / \mathrm{c}, \quad D=12 \mathrm{~cm}^{2} / \mathrm{c}, D_{p^{N^{*}}}=2,6 \mathrm{~cm}^{2} / \mathrm{c} ; b=20, W=0,008 \mathrm{~cm}$, $S_{A}=0,3 \quad \mathrm{~cm}^{2} ;$ суммарное контактное сопротивление структуры $R_{\text {cont }}=2 \cdot 10^{-4} \mathrm{Oм} \cdot \mathrm{cm}^{2} ; U_{A}=300 \mathrm{~B} ; R=0,15$ Ом.

Режим включения диода (установившееся значение тока $\sim 2 \cdot 10^{3} \mathrm{~A}$ ) был выбран специально для выявления степени влияния на переходный процесс эффектов излучательной рекомбинации и фотонной генерации, которые проявляются сильнее при больших концентрациях носителей. Как видно по кривым рис. 1 , при уменьшении $v$ переходный процесс затягивается, а падение напряжения на структуре увеличивается. Это вполне понятно, поскольку в этом случае суммарная скорость рекомбинационных процессов растет, а эффективное время жизни уменьшается. Однако для значений $v=0,9$ и $v=1$ (оптические эффекты отсутствуют) характеристики переходного процесса отличаются незначительно, а при $\boldsymbol{v}=0,99$, что соответствует экспериментальным оценкам $\left[{ }^{5}\right]$; кривая напряжения практически совпадает с кривой при $v=1$, т. е. когда 


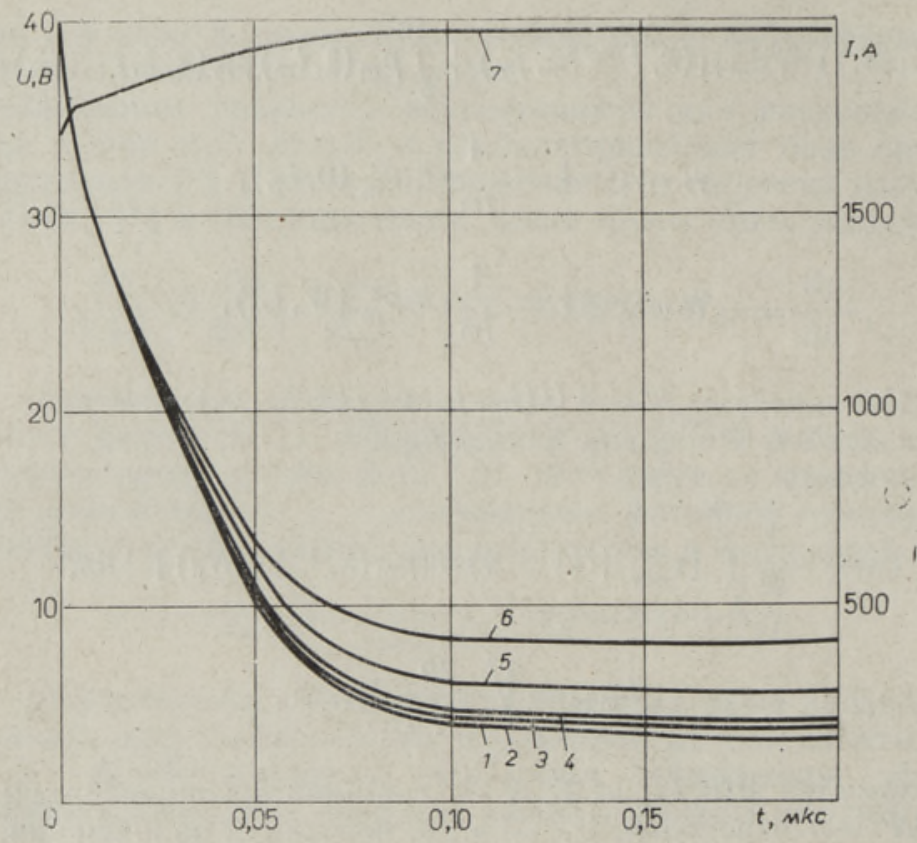

Рис. 1. Переходные характеристики напряжения $(1-6)$ и тока (7) в процессе установления стационарного состояния для $v$ : 1,0 (I), 0,99 (2), 0,9 (3), 0,8 (4), 0,5 (5), 0,0 (6).

рекомбинационные процессы определяются безызлучательным временем жизни. Это тем более справедливо для менее жестких режимов включения диода на меньшие нагрузочные токи, поскольку при этом концентрации инжектированных носителей будут меньше, а оптические эффекты выражены слабее.

Таким образом, для реальных силовых $P^{+}-N-N^{+}$-структур в ряде практически интересных режимов переключения переходные характеристики установления будут определяться величиной безызлучательного времени жизни дырок в $N$-базе, что согласуется с результатами $\left[{ }^{8}\right]$, в которой проведена оценка времени установления прямого падения напряжения в диодной структуре с «толстой» базой $(W / L>10)$. Результаты настоящей работы позволяют распространить этот вывод на структуры реальных силовых арсенид-галлиевых диодов.

Как видно из рис. 1, падение напряжения на диодной структуре составляет в начальный момент времени 40 В, тогда как исходное напряжение равно 300 В. При этом ток устанавливается практически мгновенно. Анализ показывает, что при подаче ступенчатого импульса напряжения на диодную структуру напряжение на ней очень быстро, за время максвелловской релаксации, падает до величины, определяемой в основном падением напряжения на немодулированной базовой области при токе, удовлетворяющем уравнению электрического баланса цепи (8). Поскольку уровень легирования $N$-базы в арсенид-галлиевых приборах примерно на порядок и отношение подвижностей электронов и дырок в 6-7 раз выше, чем в кремниевых диодах, а время жизни дырок на порядок меньше, установление прямого падения напряжения в арсенид-галлиевых диодных структурах происходит примерно на порядок быстрее, чем в частотных кремниевых диодах, составляя величину в несколько сотен наносекунд. 


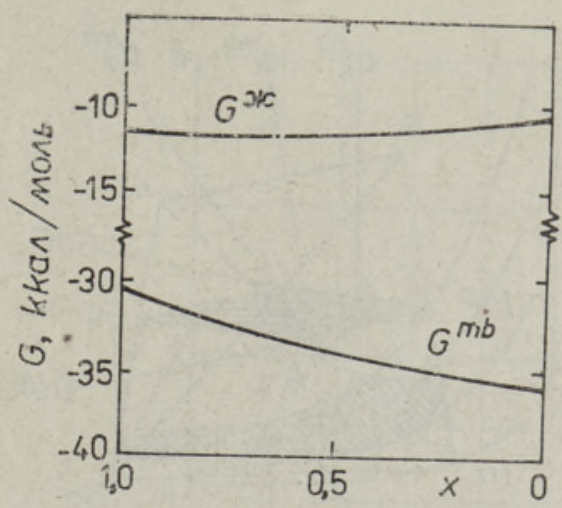

Рис. 1. Изменение свободной энергии (по Гиббсу) твердой фазы $\left(G^{\text {тв }}\right)$ в зависимости от состава твердого раствоpa $\operatorname{In}_{x} \mathrm{Ga}_{1-x}$ As. $G^{\text {ж }}$ - свободная энергия жидкой фазы, равновесной при выбранной температуре (900 К) с'твердым раствором заданного состава [ $\left.{ }^{4}\right]$.

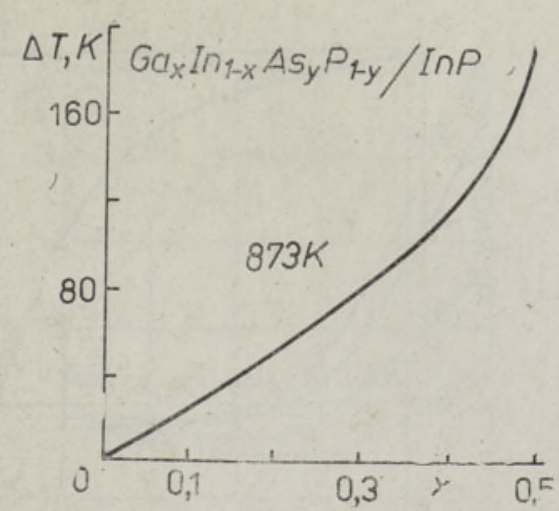

Рис. 2. Расчетная величнна пе есхлаждения $(\Delta T)$, предотвращающего растворение подложки фосфида индия при контакте с расплавом $\mathrm{Ga}-\mathrm{In}-\mathrm{As}-\mathrm{P}$ (состав жидкой фазы выражен через состав равновесного с ней твердого раствора $\left.\mathrm{Ga}_{x} \ln _{1-x} \mathrm{As}_{y} \mathrm{P}_{1-y}\right)\left[{ }^{8}\right]$.

Подложка будет стабильна при контакте с неравновесной жидкой фазой, если для химического сродства $(A)$ реакции (1) выполняется условие $\left[{ }^{9}\right]$

$$
A=\mu_{A B}^{\mathrm{TB}}-\mu_{A}^{\text {\% }}-\mu_{B}^{\%} \leqslant 0,
$$

где $\mu-$ химический потенциал. Предполагая регулярность жидкого раствора при заданном составе жидкой фазы, определялась температура $\left(T^{0}\right)$, при которой уравнение $(2)$ обращается в ноль. Оказалось, что для случая контакта различных четырехкомпонентных жидкостей с подложкой $(\mathrm{Al}-\mathrm{G} a-\mathrm{As}-\mathrm{Sb} / \mathrm{GaSb}, \mathrm{Ga}-\mathrm{In}-\mathrm{As}-\mathrm{P} / \mathrm{InP}$ и In-P-As-Sb/ InAs) $T^{0}<T^{л}$ (температура ликвидуса жидкой фазы), т. е. для предотвращения растворения подложки жидкую фазу необходимо переохлаждать на $\Delta T=T^{\pi}-T^{0}$. Величина переохлаждения зависела от состава жидкой фазы и для системы Ga-In-As-P/InP достигала значительных величин (>100 K) по мере приближения состава жидкой фазы к равновесной с твердым раствором $\mathrm{Ga}_{0,47} \operatorname{In}_{0,53}$ As (рис. 2).

Практически аналогичный подход использовали французские исследователи $\left[{ }^{10}\right]$ для анализа жидкофазной гетероэпитаксии твердого раствора $\mathrm{Ga}_{x} \mathrm{In}_{1-x} \mathrm{As}_{y} \mathrm{P}_{1-y}$ на подложках фосфида индия. При заданных составе жидкой фазы и температуре контакта они рассчитывали химическое сродство реакции (1) в зависимости от состава жидкой фазы и также констатировали, что максимальная тенденция к растворению подложки должна проявляться при гетероэпитаксии твердого раствора $\mathrm{Ga}_{0,47} \operatorname{In}_{0,53} \mathrm{As}$ (рис. 3 ).

Если для случая гетероэпитаксии твердых растворов $\mathrm{Al}_{x} \mathrm{Ga}_{1-x} \mathrm{As}_{y} \mathrm{Sb}_{1-y} / \mathrm{GaSb}_{\mathrm{InP}} \mathrm{As}_{y_{2}} \mathrm{Sb}_{1-y_{1}-y_{2}} / \mathrm{InAs}$ рассчитанные величнны переохлаждения (или, что то же самое, тенденция к растворению подложки) довольно неплохо согласуются с экспериментально установленными $\left[{ }^{11}\right]$, то для гетероэпитаксии $\mathrm{Ga}_{x} \operatorname{In}_{1-x} \mathrm{As}_{y} \mathrm{P}_{1-y} / \mathrm{InP}$ расчет значительно расходится с экспериментом. Так, эпитаксиальный рост твердого раствора $\mathrm{Ga}_{0,47} \mathrm{In}_{0,53} \mathrm{As}$ на фосфиде индия экспериментально осуществляли при очень малых переохлаждениях жидкой фазы $\left[{ }^{12}\right]$, в то время как расчетная величина $\Delta T$ превышает $100 \mathrm{~K}$.

Одной из основных причин такого несоответствия для гетероэпитаксии твердого раствора $\mathrm{Ga}_{x} \mathrm{In}_{1-x} \mathrm{As}_{y} \mathrm{P}_{1-y}$, по-видимому, является довольно быстрое установление квазиравновесия на границе раздела расплав- 


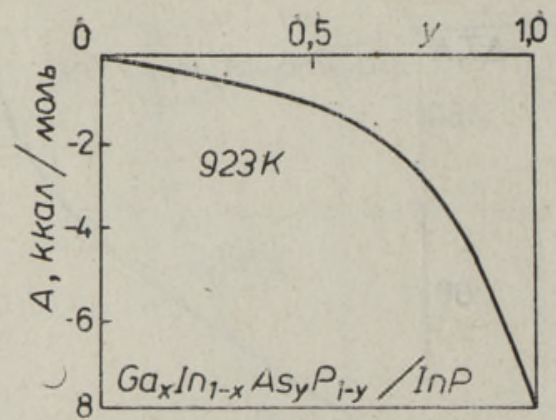

Рис. 3. Зависимость химического сродства $(A)$ для реакции (1) от состава расплава Ga-In-As-P (выражен через состав равновесного с жидкой фазой твердого раствора
$\left.\mathrm{Ga}_{x} \operatorname{In}_{1-x} \mathrm{As}_{y} \mathrm{P}_{1-y}\right)\left[{ }^{10}\right]$.

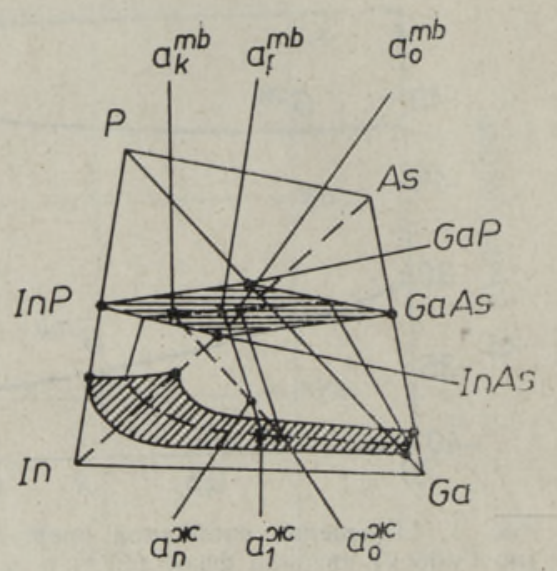

Рис. 4. Участок изотермического сечения диаграммы состояния системы $\mathrm{Ga}-\mathrm{In}-\mathrm{As}-\mathrm{P}\left[{ }^{19}\right]$.

кристалл, в результате чего подложка защищается от воздействия на нее жидкой фазы тонким слоем твердого раствора, состав которого на поверхности соответствует равновесному с расплавом.

Механизм образования такого защитного слоя получил две интерпретации. Так, М. В. Смолл и К. Дж. Гец $\left[{ }^{13}\right]$ предполагают, что квазиравновесие наступает за счет возникновения диффузионного слоя на поверхности подложки. Анализируя процесс жидкофазной гетероэпитаксии твердого раствора $\hat{A} l_{x} \mathrm{Ga}_{1-x} \mathrm{As}$ на подложках арсенида галлия, они предположили, что в процессе растворения подложки происходит диффузия алюминия из жидкой фазы в подложку. Однако при этом величина коэффициента диффузии алюминия в твердой фазе была принята на несколько порядков превышающей экспериментально установленную.

Другая группа исследователей $\left[{ }^{14}\right]$ предполагала, что образование защитного слоя происходит эпитаксиальным путем за счет смешивания двух жидкостей (тонкого слоя растворившейся подложки и основного объема расплава) в изотермических условиях.

Хотя достоверность той или иной модели до сих пор не очевидна, появившиеся в последнее время экспериментальные данные [ $\left[{ }^{15,16}\right]$ позволяют считать, что диффузия не является основным процессом при образовании защитного слоя. По-видимому, и эпитаксиальный рост и диффузия в твердой фазе имеют место при контакте неравновесных фаз, причем определяющей стадией является эпитаксиальный рост защитного слоя.

В то же время следует отметить, что защитный слой в реальных условиях жидкофазной эпитаксии изопериодных структур может на практике и не образовываться. Так, например, при больших переохлаждениях расплава индий-мышьяк-сурьма (на уровне $20 \mathrm{~K}$ ) наблюдается растворение в расплаве подложки антимонида галлия при выращивании на ней изопериодного твердого раствора InAs $\mathrm{s}_{0,9} \mathrm{Sb}_{0,1}\left[{ }^{17}\right]$.

Таким образом, для оценки устойчивости подложки относительно контактирующей с ней неравновесной жидкой фазы необходимо установить - будет ли образовываться на подложке защитный слой или нет.

Такие оценки были произведены для случая гетероэпитаксии твердого раствора $\mathrm{Al}_{x} \mathrm{Ga}_{1-x} \mathrm{As}$ на подложках $\mathrm{GaAs}\left[{ }^{18}\right]$ и для случая выращивания двойной гетероструктуры $\operatorname{InP} / \mathrm{Ga}_{x} \operatorname{In}_{1-x} \mathrm{As}_{y} \mathrm{P}_{1-y} / \operatorname{InP}\left[{ }^{19}\right] . \mathrm{Pac}-$ сматривалась модель, в которой установление квазиравновесия на границе контактирующих фаз пронсходит эпитаксиальным путем, и при за- 

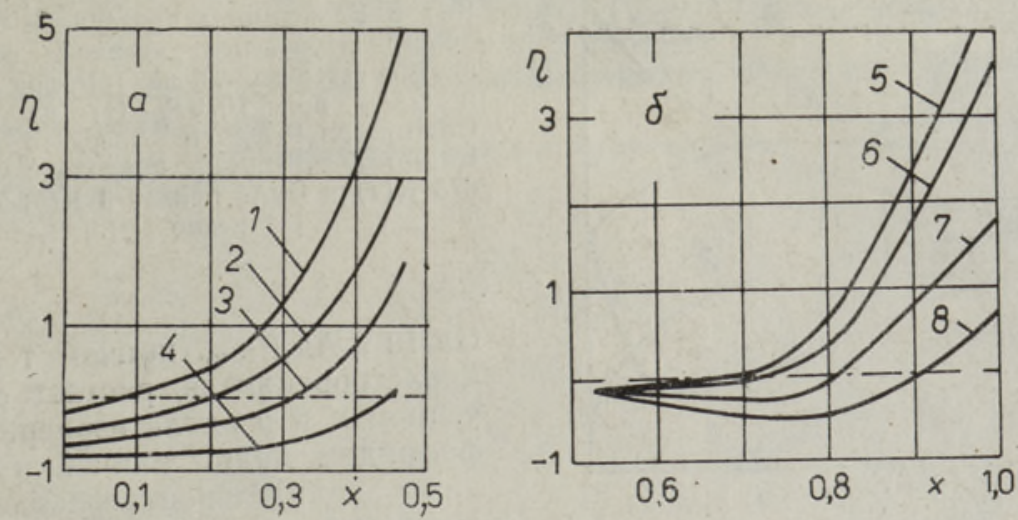

Рис. 5. Расчетные значения параметра $\eta$ для взаимодействия расплава $\mathrm{Ga}$-In-As-P (состав жидкой фазы выражен через равновесный с ней твердый раствор) с твердым раствором $\mathrm{Ga}_{x} \operatorname{In}_{1-x} \mathrm{As}_{y} \mathrm{P}_{1-y}(x ; 1-0,08$, $2-0,20,3-0,32,4-0,44,5-0,60,6-0,70,7-0,80$ и $8-0,90$; $1-4-y \simeq 2,2 \mathrm{x}, 5-8-y \simeq 2,1 \mathrm{x}-1,1)$, изопериодным $a-$ с фосфидом индия $(873 \mathrm{~K})$ и б- с арсенидом галлия (1073 K) [19].

данном количестве атомов твердой фазы, перешедших из подложки при еe растворении в расплав, рассчитывали термодинамический стимул кристаллизации, т. е. определяли какое число атомов закриста́ллизуется в результате пересыщения жидкой фазы. Сопоставление количества растворившихся и закристаллизовавшихся атомов и позволило оценить возможность образования защитного слоя,

Рассмотрим основные этапы расчета на примере гетероэпитаксии трехслойной композиции $\operatorname{InP} / \mathrm{Ga}_{x} \operatorname{In}_{1-x} \mathrm{As}_{y} \mathrm{P}_{1-y} / \operatorname{InP}\left[{ }^{19}\right]$. Пусть расплав, соответствующий по составу в концентрационном тетраэдре точке $a_{0}$ ж (рис. 4), приводится в контакт при постоянной температуре $T$ с неравновесной твердой фазой состава точки $a_{\mathrm{K}}{ }^{\text {тв }}$ (состав равновесного с расплавом $a_{0}{ }^{*}$ твердого раствора соответствует точке $\left.a_{0}{ }^{\mathrm{TB}}\right)$. При растворении части подложки состав жидкой фазы изменился (точка $a_{\text {п }}{ }^{\text {}}$ ) за счет перехода в нее атомов компонентов подложки. Поскольку при этом жидкая фаза становится пересыщенной, то из нее происходит выделение твердого раствора состава точки $a_{1}{ }^{\mathrm{TB}}$ до тех пор, пока жидкость не достигнет равновесного состава (точка $a_{1}{ }^{\text {ж}}$ ). Путем сравнения количеств растворившейся $\left(a_{\Pi}{ }^{ж} a_{0}{ }^{ж} / a_{\mathrm{K}}{ }^{\mathrm{TB}} a_{0}{ }^{ж}\right)$ и выделившейся $\left(a_{\Pi}{ }^{*} a_{1}{ }^{*} / a_{1}{ }^{\mathrm{TB}} a_{1}{ }^{*}\right)$ твердой фаз и оценивалась возможность растворения подложки или защиты ее слоем твердого раствора.

При расчете совместно решались система уравнений, определяющих фазовые границы в системе $\mathrm{Ga}-\mathrm{In}-\mathrm{As}-\mathrm{P}$, и система уравнений массового баланса при переходе атомов из одной фазы в другую и определялся параметр $\eta=\left(n_{\mathrm{K}}^{\text {Tв }}-n_{\mathrm{p}}{ }^{\mathrm{TB}}\right) / n_{\mathrm{p}}{ }^{\mathrm{TB}}\left(n_{\mathrm{p}}^{\mathrm{TB}}-\right.$ относительное количество атомов твердой фазы, перешедших в расплав при растворении подложки и $n_{\mathrm{k}}{ }^{\mathrm{TB}}$ - относительное количество атомов твердой фазы, закристаллизовавшихся из пересыщенного расплава). Положительному значению $\eta$ соответствует образование на подложке защитного слоя, а при $\eta<0$ подложка должна растворяться.

Результаты расчета показывают (рис. 5, a), что при выращивании изопериодного с фосфидом индия твердого раствора $\mathrm{Ga}_{x} \operatorname{In}_{1-x} \mathrm{As}_{y} \mathrm{P}_{1-y}$ любого состава подложка всегда будет покрываться защитным слоем твердого раствора и для получения резкой гетерограницы достаточны незначительные переохлаждения жидкой фазы. В то же время при осаждении верхнего слоя фосфида индия на твердом растворе должно происходить растворение эпитаксиального слоя твердого раствора 


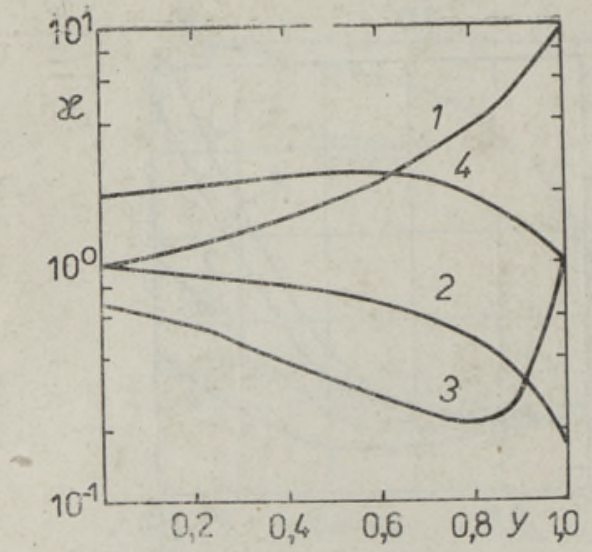

Рис. 6. Зависимость критерия $x$ от состава кристаллизующейся твердой фазы для случая гетероэпитаксин InP при 873 K(2) и $\mathrm{GaAs}$ при $1073 \mathrm{~K}$ (4) на подложке $\mathrm{Ga}_{x} \operatorname{In}_{1-x} \mathrm{As}_{y} \mathrm{P}_{1-y}$ и твердого раствора $\mathrm{Ga}_{x} \operatorname{In}_{1-x} \mathrm{As}_{y} \mathrm{P}_{1-y}$ на подложках $\operatorname{InP}$ при $873 \mathrm{~K}(1)$ и $\mathrm{GaAs}$ при $1073 \mathrm{~K}(3)$ соответственно [22].

$\mathrm{Ga}_{x} \mathrm{In}_{1-x} \mathrm{As}_{y} \mathrm{P}_{1-y}$, причем тенденция к растворению возрастает с увеличением $x$ и $y$ вдоль изопериодной с фосфидом индия линией и необходимо значительное переохлаждение жидкой фазы для предотвращения растворения твердого раствора.

Результаты расчета полностью согласуются с экспериментальными данными и объясняют трудности получения двойных гетероструктур, имеющих состав активного слоя $x \geqslant 0,40$ и $y \geqslant 0,90\left[{ }^{20}\right]$.

Аналогичным образом был проанализирован случай эпитаксии твердого раствора $\mathrm{Ga}_{x} \mathrm{In}_{1-x} \mathrm{As}_{y} \mathrm{P}_{1-y}$ на подложке арсенида галлия (рис. 5, б). Как можно видеть, в этом случае на подложке не образуется защитного слоя, что приводит к необходимости переохлаждать жидкую фазу. Расчетные оценки также согласуются с экспериментальными. Так, растворение подложки GaAs в случае осаждения на ней изопериодного твердого раствора $\mathrm{Ga}_{x} \mathrm{In}_{1-x} \mathrm{As}_{y} \mathrm{P}_{1-y}$ с малым содержанием мышьяка удавалось предотвратить только при переохлаждении расплава на $15 \mathrm{~K}\left[{ }^{21}\right]$.

Дальнейшее развитие рассмотренный подход получил в $\left[{ }^{22}\right]$. Для случая контакта $n$-компонентной жидкой фазы, содержащей $m$-компонентов типа $A$ и $(n-m)$-компонентов типа $B$, с $n$-компонентной подложкой устойчивость подложки оценивается с помощью критерия $\varkappa\left(\varkappa=n_{\mathrm{K}}{ }^{\mathrm{TB}} / n_{\mathrm{p}}{ }^{\mathrm{TB}}\right)$

$$
x=\frac{\sum_{i} k_{i} c_{i}^{\mathrm{II}}}{\sum_{i} k_{i} c_{i}}
$$

Здесь $k_{i}-$ коэффициент распределения $i$-го компонента, $c_{i}$ и $c_{i}$ п - стехиометрическне коэффициенты в равновесном с расплавом твердом растворе и в подложке соответственно $\left(x_{i}=c_{i}\left(c_{i}{ }^{\mathrm{I}}\right)\right.$ при $1 \leqslant i \leqslant m$ и $y_{i}=c_{i}\left(c_{i}\right.$ п) при $\left.m<i \leqslant n\right)$. Квазиравновесие на границе контактирующих неравновесных фаз устанавливается, по мнению авторов $\left[{ }^{22}\right]$, путем образования эпитаксиального слоя за счет смешения атомов подложки, перешедших в жидкую фазу, и основного объема расплава, причем состав эпитаксиального слоя изменяется от соответствующего подложке до равновесного с расплавом твердого раствора. С помощью довольно просто определяемого критерия (3) были проанализированы условия гетероэпитаксии четырехкомпонентных твердых растворов, изопериодных с подложками соединений типа $\mathrm{A}^{\mathrm{II}} \mathrm{B}^{\mathrm{V}}$. и показано, что при гетероэпитаксии $\mathrm{Al}_{x} \mathrm{Ga}_{1-x} \mathrm{As}_{y} \mathrm{Sb}_{1-y} / \mathrm{GaSb}, \mathrm{Ga}_{x} \mathrm{In}_{1-x} \mathrm{As}_{y} \mathrm{Sb}_{1-y} / \mathrm{GaSb}, \mathrm{InP}_{y_{1}} \mathrm{As}_{y_{2}} \mathrm{Sb}_{1-y_{1}-y_{2}} /$ InAs подложка должна растворяться, и тогда необходимо переохлаждение расплава для инициирования кристаллизации твердого раствора, подавляющей растворение подложки. Для случая гетероэпитаксии InP и GaAs на подложку $\mathrm{Ga}_{x} \mathrm{In}_{1-x} \mathrm{As}_{y} \mathrm{P}_{1-y}$ и для обратной ситуации результаты расчета соответствуют $\left[{ }^{19}\right]$ (рис. 6). Максимальная тенденция к растворению подложки среди трех типов гетероструктур выявлена для композиций $\mathrm{Ga}_{x} \mathrm{In}_{1-x} \mathrm{As}_{y} \mathrm{Sb}_{1-y} / \mathrm{GaSb}$ и $\mathrm{Al}_{x} \mathrm{Ga}_{1-x} \mathrm{As}_{y} \mathrm{Sb}_{1-y} / \mathrm{GaSb}$. Этим, по- 


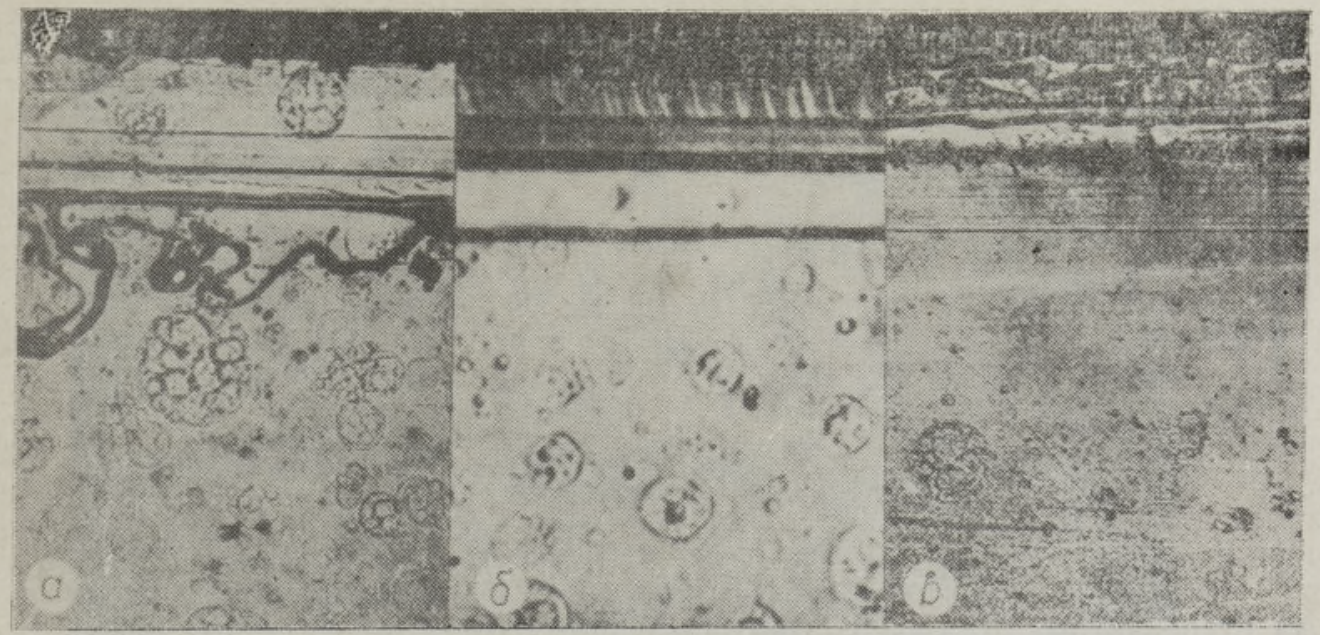

Рис. 7. Микрофотографии поперечных сколов, иллюстрирующие морфологию гетероструктур на основе GaAlAsSb/GaSb, изготовленных методом жидкофазной эпитаксии: $a$ - без переохлаждения расплава, с подрастворением подложки; б - с переохлаждением при выращивании буферного слоя; в - то же при выращивании на подложке, разориентированной на $3^{\circ}$ от плоскости $(100)\left[{ }^{23}\right]$.

видимому, и объясняется образование т. н. языков проплавления при выращивании гетероструктуры $\mathrm{Al}_{x} \mathrm{Ga}_{1-x} \mathrm{As}_{y} \mathrm{Sb}_{1-y} / \mathrm{GaSb}$ (рис. 7) [23]. Поскольку растворение подложки вероятнее всего происходит по дефектам, и количество образовавшейся при пересыщении твердой фазы значительно меньше растворившейся, то возможно «замуровывание» расплава выпадающим в верхней части вытравленного объема твердым раствором. Неравновесность «замурованной» жидкости с подложкой и приводит к растворению подложки и движению капли в подложку.

Таким образом, при гетероэпитаксии из жидкой фазы могут встретиться случай устойчивости подложки или ее растворения при контакте с неравновесной жидкой фазой. В первом случае эпитакснальный рост может осуществляться при очень незначительном переохлаждении жидкой фазы. В связи с этим в последнее время получил развитие вариант «равновесного охлаждения» для выращивания эпитаксиальных слоев $\mathrm{Ga}_{x} \operatorname{In}_{1-x} \mathrm{As}_{y} \mathrm{P}_{1-y}$, изопериодных с фосфидом индия (устойчивая подложка), в котором расплав не переохлаждается $(\Delta T=0)$ перед контактом c подложкой. В то же время предотвращение растворения $\mathrm{Ga}_{x} \mathrm{In}_{1-x} \mathrm{As}_{y} \mathrm{P}_{1-y}$ (растворяющаяся подложка) при осаждении на нем фосфида индия требует переохлаждение $10-12 \mathrm{~K} \quad\left[{ }^{24}\right] \quad(x \simeq 0,40$, $y \simeq 0,90$ ).

В общем случае оценка требуемой величины переохлаждения расплава для сдучая растворяющейся подложки может производиться по методике $\left[{ }^{8}\right]$.

Однако не всегда возможно использовать переохлаждение расплава для предотвращения растворения подложки. Обычно величина требуемого переохлаждения $\Delta T \geqslant 5-8 \mathrm{~K}$, однако при этом возможно изменение состава кристаллизующегося твердого раствора, а следовательно, и величины рассогласования периодов решеток на гетерогранице $(\Delta a)$. Кроме того, возможно образование гомогенно зародившихся кристалликов твердого раствора, также изменяющих состав эпитаксиально выращиваемого твердого раствора. .

Отмеченные обстоятельства и приводят к зависимости скорости роста твердых растворов $\mathrm{Ga}_{x} \mathrm{In}_{1-x} \mathrm{As}_{y} \mathrm{Sb}_{1-y}$ от величины переохлаждения (рис. 8). Увеличение $\Delta a$ с возрастанием $\Delta T$ и приводит к снижению ско- 


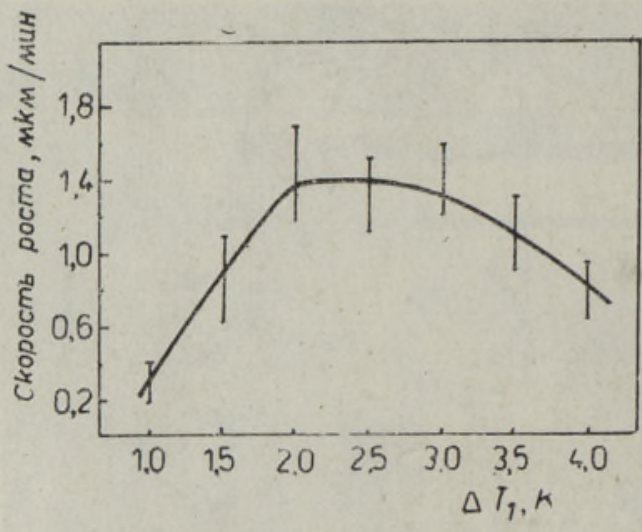

Рис. 8. Зависимость скорости роста слоев $\mathrm{GaInAsSb/GaSb}$ от величины переохлаждения раствора-расплава. Температура начала кристаллизацин слоев $724 \mathrm{~K}$.

рости роста слоев после достижения некоторой оптимальной величины $\Delta T$. Зависимость состава твердых растворов $\mathrm{Al}_{x} \mathrm{Ga}_{1-x} \mathrm{As}_{y} \mathrm{Sb}_{1-y}$ и $\mathrm{Ga}_{x} \mathrm{In}_{1-x} \mathrm{As}_{y} \mathrm{Sb}_{1-y}$ от .величины переохлаждения и не позволяет использовать для их эпитаксиального выращивания метода ступенчатого охлаждения, который широко используется для получения $\mathrm{Ga}_{x} \mathrm{In}_{1-x} \mathrm{As}_{y} \mathrm{P}_{1-y}$ на подложках $\mathrm{InP} c$ ориентацией (100), поскольку состав такого твердого раствора практически не изменяется при $\Delta T=10 \mathrm{~K}$ и не происходит объемной кристаллизации. Возможным вариантом получения планарной гетерограницы в случае композиций

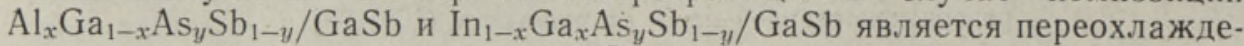
ние не жидкой фазы, а подложки $\left[{ }^{17}\right]$.

Обсужденные выше контактные явления ставят под сомнение как фазовые границы в четырехкомпонентных системах, полученные различными авторами, так и определенные по ним термодинамические параметры, используемые при расчетных оценках равновесных составов жидкой и твердой фаз $\left[{ }^{25}\right]$. Поэтому всякие «уточнения» диаграмм состояния, а особенно поверхности ликвидуса методом насыщения (см. $\left[{ }^{26}\right]$ ), на наш взгляд, беспредметны, поскольку устанавливаются при этом только очередные «эффективные» фазовые границы.

Как уже отмечалось, помимо термодинамической нестабильности контактирующих фаз на эпитаксиальный рост оказывает влияние и поверхностная кинетика, которая требует учета особенностей осаждения при различной ориентации подложки.

Кристаллизация эпитаксиального слоя в условиях минимального травления металлическим расплавом материала подложки предъявляет повышенные требования как к подготовке материала подложки для эпитаксии и состоянию ее поверхности на стадии, предшествующей эпитаксиальному росту, так и к заданной ориентации поверхности подложки. В этой связи при ЖФЭ изопериодных структур на основе четверных твердых растворов было обращено $\left[{ }^{27}\right]$ серьезное внимание на предотвращение термического травления подложки на стадии предэпитаксии и отчасти на предотвращение термического травления поверхности структуры во время роста. Сказанное особенно важно при выращивании структур $\mathrm{Ga}_{x} \mathrm{In}_{1-x} \mathrm{As} \mathrm{s}_{y} \mathrm{P}_{1-y} / \mathrm{InP}$, так как термическое травление подложки фосфида индия происходит в некоторой степени даже при температурах $650-700 \mathrm{~K}$ (у антимонида галлия - лишь при температурах более 900 K). Характерные фигуры термического травления поверхности подложки показаны на рис. 9. Достаточно сложно избежать полного отсутствия этих фигур на поверхности подложки, так как при превышении давления фосфора выше требуемого уровня (создается обычно испарением фосфора из расплава олова) на поверхности подложки могут образоваться включения второй фазы. В этой связи принципиальным оказывается направление перемещения расплава и подложки относительно друг друга. Для наиболее распространенной (при жидкофазной эпитаксии рассматриваемых материалов) ориентации подложки (100) таким направлением является [011]. Указанное направление может быть опре- 


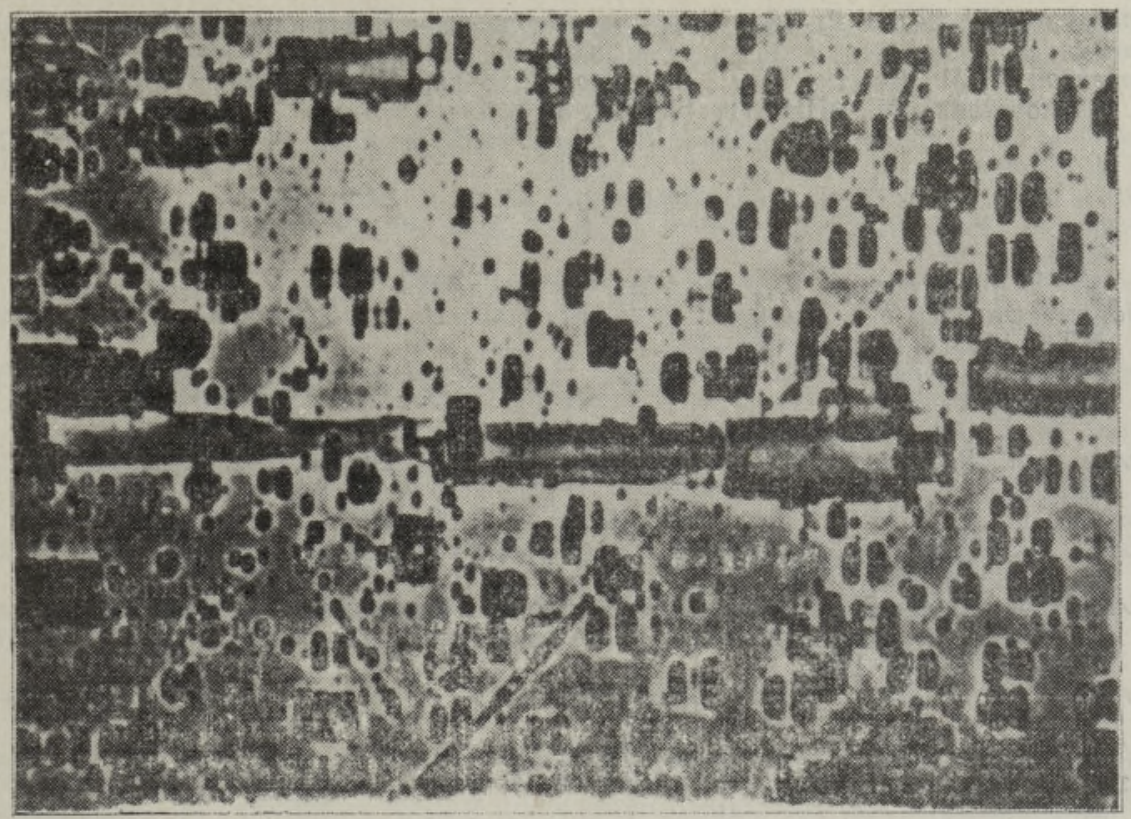

Рис. 9. Фигуры химического травления (вытянуты в направлении [011]) и термического травления (вытянуты в направлении [011]) на поверхности (100) подложки InP.

делено по ориентировке фигур травления на поверхности (100) (рис. 9). Вытянутая сторона этих фигур соответствует направлению [011]. Таким образом, по тестовым образцам, отколотым от подложки, определяют (после их химичческого травления) требуемое направление перемещения подложки. Сформулированные здесь представления наиболее важны при росте слоев на подложке, поверхность (100) которой подверглась термическому разложению, даже незначительному. Как показывают опыты, если перемещать подложку, например, в направлении [011], перпендикулярном вытянутой стороне фигур термического травления, то в процессе эпитаксии не удается избежать капель металла на поверхности слоя (структуры), в то время как при перемещении подложки в направлении [011] металлических капель не наблюдается.

Мы более подробно остановились на последствиях отсутствия термодинамического равновесия в системе подложка-расплав при ЖФЭ изопериодных структур, так как оно, как нами было показано, не только оказывает существенное влияние на характер контактных явлений при начальных стадиях роста слоев, но и во многом определяет выбор конкретного способа изготовления гетероструктур.

\section{Л И ТЕ Р А Т Р А}

1. Hsieh, J. J. J. Cryst. Growth. 1974. 27, 49-61.

2. Долгинов Л. М., Елисеев П. Г., Мильвидский М. Г. Квантовая электроника, 1976, 3, № 7, 1381-1392.

3. Blom, G. M. J. Electrochem. Soc., 1971, 118, № 11, 1834-1936.

4. Astles, M. G., Dosser, O. D., Mac Lean, A. J., Wright, P. J. J. Cryst. Growth, 1981,54, № $3,485-492$.

5. Глазов В. М., Павлова Л. М. Химическая термодинамика и фазовые равновесия. М., «Металлургия», 1981.

6. Dorfman. V. F.. Shupegin, M. L. J. Cryst. Growth. 1981, 52, № ? 710-715.

7. Болховитянов Ю. Б. Препринт 2-82. Новосибирск, ИФП СО АН СССР, 1982.

8. Вигдорович В. Н., Селин А. А., Шутов С. Г., Батура В. П. Изв. АН СССР. Неорган. матер., 1981,17 , № 1, 10-13, 
9. Пригожин И., Дефэй Р. Химическая термодинамика. Новосибирск, «Наука», 1966.

10. Quillec, M., Benchimol, J. L. J. Cryst. Growth. 1981, 54, № 1, 76-80.

11. Долгинов Л. М., Елисеев П. Г., Нсмаилов И. В кн.: Итоги науки и техники. Сер. радиотехника. М., ВИНИТИ, 1980, 21, 3-115.

12. Nakajima, K., Tanahashi, T., Akita, K., Yamaoka, T. J. Appl. Phys., 1979, 50, № 7 , $4975-4981$.

13. Small, M. B., Ghez, R. J. Appl. Phys, 1979, 50; № 8, 5322-5330.

14. Kordos, P., Pearson, G. L., Panish, M. B. J. Appl. Phys, 1979, 50, № 11, 6902 6906.

15. Petroff, P. M., Logan, R. A. J. Vac. Sci. Technol,, 1980, 17, № 5, 1113--1117.

16. Абдурахманов Ю. Ю., Клименко С. Е., Корсуков В. Е., Яковлев Ю. П. Письма в ЖТФ, 1982, 8, вып. 12, 762-765.

17. Gertner, E. R., Andrews, A. M., Babulac, L. O. et al. J. Electron. Materials, 1979, 8, № 4, 545-554.

18. Small, M. B., Ghez, R. J. Appl. Phys., 1980, 51, № 3, $1589-1592$.

19. Селин А. А., Вигдорович В. П., Батура В. П. Ж: физ. химии, 1982, 56, № 6, $1360-$ 1364.

20. Houston, P. A. J. Mater. Sci., 1981, 16, № 1, 2935-2961.

21. Suzuki, A., Kyuragi, H., Matsumura, S., Matsunami, H. Jap. J. Appl. Phys., 1980, 19, № 4, 207-210.

22. Селин A. А., Ханин В. А. В кн.: Тез. докладов VI конференции по процессам роста и синтеза полупроводниковых кристаллов и пленок. Новосибирск, ИНХ СО АН CCCP, 1, 92-93, 1981

23. Aarik, J. A.; Dolginov, L. M., Druzhinina, L. V. et al. Krist. und Techn., 1980, 15, № 11, 1311-1316.

24. Hsieh, J. I. Appl. Phys. Lett., 1980. 37, № 1, 25-27.

25. Батура В. П., Вигдорович В. Н., Селин А. А. Зарубеж. электрон. техника, 1980, № $12,3-52$.

26. Bert, N. A., Gorelenok, A. T., Dzigasov, A. G. et al. J. Cryst. Growth, 1981, 52, № 2, $716-721$.

27. Долгинов Л. М., Жукова Л. А., Малькова Н. В., Югова Т. Г. Электронная техника. Сер. 6. Материалы, 1981, вып. 10, 27-29.

\section{Государственный научно-исследовательский \\ и проектный институт редкометаллической промышленности}

\section{Ннститут физики}

Академии наук Эстонской ССР

Московский иніститут электронной техники

L. DOLGINOV, L. DRUZININA, P. LOUK.

A. SELIN, J. FRIEDENTHAL

\section{KONTAKTNÄHTUSED VEDELIKEPITAKSIAS}

Artiklis on kirjeldatud vedelikepitaksias toimuvaid lontaktnähtusi ja näidatud, et termodünaamilise tasakaalu puudumine süsteemis binarrne alıs-paliukomponendiline sulamlahus määrab nii kristallisatsiooni algstaadiumi kui ka konkreetsed protsessi läbiviimise tingimused.

\section{DOLGINOV, L. DRUZHININA, P. LOUK.}

\section{A. SELIN, J. FRIEDENTHAL}

\section{THE CONTACT PHENOMENA IN LIQUID PHASE EPITAXY}

The phenomena taking place at the interface of jiquid and solid phases in liquid phase heteroepitaxy of the latticematched $\mathrm{III}-\mathrm{V}$ compound semiconductor solid solutions are discussed in detail. It is shown that under isothermal conditions, because of the lack of thermodynamical equilibrium between the liquid and solid phases, a partial or complete dissolution of the substrate takes place. To avoid this process, it is necessary to usz the supercooling of the liquid phase by a certain value of $\Delta T$. Numerical values of $\Delta T$ are compared with experimental results for many systems. It is shown that in some cases quasiequilibrium can be reached very rapidily at the interface as the solid surface reaches a concentration very close to that of equilibrium. A comparison of the numbers of crystallized and dissolved atoms, as is shown for the case of the $\operatorname{In} \mathrm{P} / \mathrm{GaInAsP} / \mathrm{InP}$ structure, enables one to predict the possibility of the formation of a protecting layer. It has been established that the lack of equilibrium defines the character of the contact phenomena not only at the first stage of the layer growth but it determines the whole process of the epitaxial growth, 